\title{
Características clínicas, de laboratorio, microbiológicas y ecocardiográficas de la endocarditis infecciosa en un hospital de tercer nivel
}

\author{
Clinical, laboratory, microbiological and \\ echocardiographic characteristics of infective \\ endocarditis in a tertiary care hospital
}

\author{
Ana G. Múnera-Echeverri, Carolina Saldarriaga-Acevedo • Medellín (Colombia)
}

DOI: https://doi.org/10.36104/amc.2021.1930

\section{Resumen}

Objetivos: la endocarditis infecciosa (EI) es una enfermedad potencialmente fatal. En el presente estudio se analizan las características clínicas, de laboratorio, microbiológicas y ecocardiográficas de la EI en una población de pacientes de un hospital de tercer nivel en Medellín-Colombia durante un periodo de tres años.

Métodos: estudio observacional retrospectivo. Los pacientes fueron clasificados de acuerdo con los criterios de Duke modificados. Se recolectaron datos clínicos, ecocardiográficos, resultados de laboratorio y cultivos de las historias clínicas. Se determinaron factores asociados con pronóstico.

Resultados: se incluyeron un total de 48 casos, 29 (60.4\%) fueron hombres. La edad media fue de $53.8 \pm 19.2$ años. La fiebre y fatiga fueron las manifestaciones clínicas más comunes. En $52.1 \%$ de los pacientes no se reportaba la presencia de soplo cardiaco al ingreso. La mayoría de pacientes $(62.5 \%)$ no tenían una condición cardiaca predisponente de base. La EI ocurrió en válvula nativa en 36 pacientes $(75 \%)$. La válvula mitral fue el sitio más afectado. La ecocardiografía transtorácica y/o transesofágica mostró vegetaciones en 45 casos (93.7\%), la mayoría móviles, con tamaño promedio de 17.6 $\pm 11.3 \mathrm{~mm}$. El Staphylococcus aureus fue el microorganismo causante principal (33\%). La prevalencia de EI con hemocultivos negativos fue del 37.5\%. La complicación más frecuente fue el embolismo en 21 pacientes (43.7\%), seguido por la falla cardiaca (41.7\%). Por análisis multivariado el choque séptico, la falla renal, la infección por Staphylococcus y el uso de inmunosupresores fueron predictores de mayor mortalidad intrahospitalaria.

Conclusiones: la mayoría de casos ocurren en pacientes de edad avanzada sin una condición cardiaca predisponente, en válvula nativa, con predilección por la válvula mitral. El Staphylococcus aureus es el microorganismo causante más frecuente. Varios factores predicen mayor mortalidad intrahospitalaria incluyendo la presencia de choque séptico, falla renal, infección por Staphylococcus y uso de inmunosupresores. (Acta Med Colomb 2021; 46. DOI: https://doi.org/10.36104/amc.2021.1930).

Palabras clave: endocarditis, ecocardiografía, epidemiología, válvulas cardíacas, Staphylococcus.

\section{Abstract}

Objectives: infective endocarditis (IE) is a potentially fatal disease. This study analyzed the clinical, laboratory, microbiological and echocardiographic characteristics of IE in a population of patients at a tertiary care hospital in Medellín, Colombia, over a three-year period.

Methods: a retrospective observational study. The patients were classified according to the modified Duke criteria. Clinical and echocardiographic data, laboratory results and cultures were gathered from the clinical charts. Factors associated with the prognosis were determined.

Results: a total of 48 cases were included, 29 (60.4\%) of which involved males. The mean age was $53.8 \pm 19.2$ years. Fever and fatigue were the most common clinical signs. No heart murmur
Dra. Ana G. Múnera-Echeverri: Internista, Cardióloga, Ecocardiografista. Servicio de Cardiología No Invasiva Hospital General de Medellín. Investigador Grupo HGM y Docente Universidad CES; Dra. Carolina SaldarriagaAcevedo: Cardióloga, Fellow de Ecocardiografía Universidad CES. Medellín (Colombia). Correspondencia: Dra. Ana G. Múnera-Echeverri. Medellín (Colombia).

E-Mail: anagm@une.net.co

Recibido: 26/VI/2020 Aceptado:19/V/2021 
was reported on admission in $52.1 \%$ of the patients. Most of the patients $(62.5 \%)$ had no underlying predisposing heart condition. The IE occurred in a native valve in 36 patients $(75 \%)$, with the mitral valve being the most frequently affected site. Transthoracic and/or transesophageal echocardiography showed vegetations in 45 cases $(93.7 \%)$; these were mostly mobile, with an average size of $17.6 \pm 11.3 \mathrm{~mm}$. Staphylococcus aureus was the main causal organism (33\%). The prevalence of IE with negative blood cultures was $37.5 \%$. The most frequent complication was embolism in 21 patients $(43.7 \%)$, followed by heart failure $(41.7 \%)$. On multivariate analysis, septic shock, kidney failure, Staphylococcus infection and the use of immunosuppressants were predictors of higher inpatient mortality.

Conclusions: most cases occur in elderly patients with no underlying predisposing heart condition, in a native valve, with a predilection for the mitral valve. Staphylococcus aureus is the most frequent causal organism. Several factors predict greater inpatient mortality, including the presence of septic shock, kidney failure, Staphylococcus infection and the use of immunosuppressants. (Acta

Med Colomb 2021; 46. DOI: https://doi.org/10.36104/amc.2021.1930)

Keywords: endocarditis, echocardiography, epidemiology, heart valves, Staphylococcus.

\section{Introducción}

La endocarditis infecciosa (EI) es una enfermedad difícil y compleja. A pesar de los avances en el diagnóstico, tratamiento médico y quirúrgico, la EI sigue asociándose con alta mortalidad y complicaciones severas (1).

El perfil epidemiológico de la EI ha cambiado sustancialmente en los últimos años $(2,3)$. Históricamente afectaba pacientes jóvenes con enfermedad valvular predisponente de base, predominantemente de origen reumático y el Streptococcus viridans era el patógeno más frecuente (4). En los últimos años la edad de los pacientes con EI ha aumentado y la valvulopatía degenerativa es la lesión predisponente más frecuente en países desarrollados donde la fiebre reumática ha dejado de ser un problema de salud pública; también ha aumentado de manera notable el número de pacientes con EI sin valvulopatía conocida previamente (5). La microbiología también ha cambiado, muchos estudios recientes indican que el Staphylococcus aureus es actualmente el principal microorganismo causante de la EI $(6,7)$.

Numerosos estudios han evaluado las características clínicas, microbiológicas y ecocardiográficas de la EI en población occidental. Sin embargo, poco es conocido sobre la epidemiología de la EI en nuestro medio, las características ecocardiográficas, los agentes microbiológicos principalmente implicados, las manifestaciones clínicas más comunes y las complicaciones en nuestros pacientes. Se hace necesario observar el comportamiento local de esta entidad para evaluar si existe algún cambio en el perfil clínico que nos pueda servir como base para el desarrollo de futuras estrategias de manejo.

En el presente estudio se analizan las características clínicas, de laboratorio, microbiológicas y ecocardiográficas de la EI en una población de pacientes de un hospital de tercer nivel en Medellín-Colombia durante un periodo de tres años.

\section{Material y métodos}

El estudio fue diseñado como un estudio observacional retrospectivo. Se incluyeron todos los pacientes mayores de
18 años con diagnóstico de EI por historia clínica evaluados en el servicio de ecocardiografía del Hospital General de Medellín entre marzo de 2011 y junio de 2014.

Se recolectaron los datos de las historias clínicas sobre las características demográficas, edad, género, enfermedad cardiaca de base, signos y síntomas de presentación, comorbilidades, resultados de laboratorios y microbiológicos, hallazgos ecocardiográficos, requerimiento quirúrgico y complicaciones.

Todos los pacientes cumplían con los siguientes requisitos: 1. Mayores de 18 años, 2. Diagnóstico definitivo o probable de EI de acuerdo con los criterios de Duke modificados, 3 . Ecocardiografía bidimensional transtorácica y/o transesofágica y 4 . Hemocultivos seriados.

\section{Definición de términos}

1. De acuerdo con los criterios de Duke modificados:

\section{Criterios mayores}

- Hemocultivos positivos: microorganismo típico consistente con EI de dos hemocultivos separados, o microorganismo consistente con EI con hemocultivos persistentemente positivos, o un hemocultivo positivo para Coxiella burnetti o títulos de anticuerpos $\operatorname{IgG}>$ 1:800.

- Evidencia de compromiso endocárdico por ecocardiografía con la presencia de vegetaciones, absceso, fístula, pseudoaneurisma o nueva dehiscencia de una prótesis valvular; actividad anormal alrededor de una prótesis valvular por PET/CT (tomografía por emisión de positrones) o SPECT/CT (tomografía computarizada con emisión de fotón único) o lesión paravalvular demostrada en una tomografía computarizada cardiaca.

\section{Criterios menores}

- Condiciones predisponentes como enfermedad cardiaca de base o uso de drogas intravenosas, fiebre, fenómenos vasculares, fenómenos inmunológicos y hemocultivos 
positivos que no clasifican como criterio mayor.

EI definitiva: en presencia de dos criterios mayores, o un mayor y tres menores, o cinco menores.

EI posible: en presencia de un criterio mayor y un criterio menor, o tres criterios menores.

\section{Seguridad y consideraciones éticas}

Según el artículo 11 de la Resolución 008430 de 1993 emitida por el Ministerio de Salud de la República de Colombia, este proyecto se clasifica como una investigación sin riesgo ya que se emplearán técnicas y métodos documentales retrospectivos; no se realizará ninguna intervención intencional de las variables fisiológicas, psicológicas, biológicas ni sociales de los individuos debido a que la fuente de la información será la historia clínica, cumpliendo a su vez con la Resolución 8430 de 1993, promulgada por el Ministerio de Salud, por la cual se establece las normas científicas, técnicas y administrativas para la investigación en salud.

La información acerca de cada una de las variables de los pacientes se digitó en una hoja de cálculo de Microsoft Excel. El análisis estadístico de los resultados se realizó con el software SPSS versión 21. Las variables cuantitativas se presentan con las medidas de tendencia central (promedio) y de dispersión (desviación estándar). Las variables categóricas se presentan como proporciones. El análisis bivariado incluyó la prueba de chi-cuadrado para las variables categóricas y la prueba $t$ de Student o U de Mann Whitney para variables cuantitativas. El nivel de confianza para las pruebas de hipótesis es $95 \%$. La asociación entre el tamaño de las vegetaciones y las complicaciones se evaluó mediante la prueba de chi-cuadrado. Para el análisis de los factores asociados con la mortalidad intrahospitalaria se realizó una regresión logística binaria, por un método por pasos hacia adelante. Para todos los análisis estadísticos un valor de $\mathrm{p}$ inferior a 0.05 se consideró estadísticamente significativo.

\section{Resultados}

Entre los 48 casos incluidos, 46 pacientes (95.8\%) fueron clasificados como endocarditis definitiva y dos pacientes (4.2\%) como endocarditis probable de acuerdo con los criterios de Duke modificados.

Las principales características de los pacientes se muestran en la Tabla 1; 29 pacientes $(60.4 \%)$ fueron hombres y 19 pacientes $(39.6 \%)$ mujeres, con una relación hombre: mujer de 1.5:1. La edad promedio ( \pm DS) fue de 53.8 \pm 19.2 años.

La endocarditis de válvula nativa ocurrió en 36 pacientes (75\%), de válvula protésica en tres pacientes $(6.3 \%)$ y asociada a dispositivo intracardiaco en nueve pacientes (18.8\%). Según el modo de adquisición de la infección 20 pacientes (42\%) tuvieron una endocarditis asociada al cuidado de la salud y 28 pacientes (58\%) endocarditis adquirida en la comunidad. La mayoría de pacientes no tenían una condición cardiaca predisponente de base $(62.5 \%)$.

Fiebre y fatiga fueron las manifestaciones clínicas más comúnmente presentadas por los pacientes (70.8 y $77.1 \%$
Tabla 1. Características basales de los pacientes con endocarditis infecciosa (EI).

\begin{tabular}{|c|c|}
\hline Variables & $\mathrm{n}=48$ \\
\hline \multicolumn{2}{|l|}{ Características basales al diagnóstico } \\
\hline Sexo masculino, $n(\%)$ & $29(60.4)$ \\
\hline Edad, X (DS) & $53.8(19.2)$ \\
\hline \multicolumn{2}{|l|}{ Comorbilidades, n (\%) } \\
\hline Hipertensión arterial & $24(50)$ \\
\hline Diabetes mellitus & $8(16.7)$ \\
\hline Enfermedad renal crónica & $9(18.8)$ \\
\hline Malignidad & $4(8.3)$ \\
\hline Previa EI & $1(2.1)$ \\
\hline Enfermedad valvular & $8(16.6)$ \\
\hline Insuficiencia aórtica & $2(4.1)$ \\
\hline Insuficiencia mitral & $4(8.3)$ \\
\hline Prolapso valvular mitral & $1(2.1)$ \\
\hline Doble lesión mitral & $1(2.1)$ \\
\hline Cardiopatía congénita & $7(14.6)$ \\
\hline Aorta bivalva & $1(2.1)$ \\
\hline CIV & $3(6.2)$ \\
\hline DAP & $1(2.1)$ \\
\hline Membrana subaórtica & $1(2.1)$ \\
\hline Canal AV & $1(2.1)$ \\
\hline Prótesis valvular & $3(6.3)$ \\
\hline Biológica & $3(6.3)$ \\
\hline Mecánica & $0(0)$ \\
\hline Dispositivo intracardiaco & $6(12.5)$ \\
\hline Enfermedades inflamatorias & $7(14.5)$ \\
\hline Uso de drogas intravenosas & $0(0)$ \\
\hline VIH & $1(2.1)$ \\
\hline Consumo esteroides u otros inmunosupresores & $6(12.5)$ \\
\hline
\end{tabular}

respectivamente), en $47.9 \%$ de los pacientes se reportó soplo cardiaco como hallazgo clínico (Tabla 2).

Hubo 30 casos $(62.5 \%)$ con hemocultivos positivos y $18(37.5 \%)$ con hemocultivos negativos, de estos últimos un paciente tuvo diagnóstico microbiológico con cultivo de tejido valvular.

La Tabla 3 resume los microorganismos causantes de la infección durante el estudio. El Staphylococcus fue la causa en $39.6 \%$ de los casos y el Streptococcus en $14.5 \%$.S. aureus 
Ana G. Múnera-Echeverri y col.

Tabla 2. Manifestaciones clínicas de los pacientes con endocarditis infecciosa.

\begin{tabular}{|l|c|}
\hline \multicolumn{1}{|c|}{ Manifestaciones clínicas } & $\mathbf{n}(\%)$ \\
\hline Fiebre & $34(70.8)$ \\
\hline Fatiga & $37(77.1)$ \\
\hline Disnea & $25(52.1)$ \\
\hline Escalofríos & $31(64.6)$ \\
\hline Convulsiones/focalización neurológica & $5(10.4)$ \\
\hline Síntomas gastrointestinales & $15(31)$ \\
\hline Dolor torácico & $6(12.5)$ \\
\hline Cefalea & $8(16.7)$ \\
\hline Pérdida de peso & $12(25)$ \\
\hline Síntomas musculares/articulares & $21(43.8)$ \\
\hline Lesiones cutáneas & $15(31.3)$ \\
\hline Soplo & $23(47.9)$ \\
\hline
\end{tabular}

fue aislado en 16 pacientes (33\%) y fue el microorganismo más frecuente, seguido por el Streptococcus sanguis en tres pacientes $(6.3 \%)$.

El diagnóstico inicial se realizó por ecocardiografía transesofágica en 26 pacientes (54.2\%) y por ecocardiografía transtorácica en 22 (45.8\%). El tiempo medio entre la sospecha clínica y la primera ecocardiografía fue de 24 horas (15-48 horas). Cuarenta y un pacientes requirieron solo una ecocardiografía para el diagnóstico, siete pacientes requirieron dos ecocardiografías para el diagnóstico. Los principales hallazgos ecocardiográficos se resumen en la Tabla 4. Se encontraron vegetaciones en 45 de los 48 pacientes (93.7\%), la mayoría móviles $(40 / 45,88.8 \%)$, la válvula mitral fue la localización más frecuente $(17 / 45,37.7 \%)$ seguida por la válvula aórtica $(16 / 45,35.5 \%)$. El tamaño promedio de las vegetaciones fue de $17.6 \pm 11.3 \mathrm{~mm}$. La complicación más frecuente fue el embolismo en 21 pacientes (43.7\%), seguido por la falla cardiaca en $41.7 \%$. Los sitios más comunes de embolismo fueron pulmón y sistema nervioso central (SNC) (9 y 8 casos, respectivamente) (Tabla 5).

Se encontró una asociación estadísticamente significativa entre infección por Staphylococcus y la presentación de embolismo pulmonar ( $\mathrm{p}=0.01)$, no así con embolismo a $\mathrm{SNC}(\mathrm{p}=0.390)$.

No se encontró una asociación estadísticamente significativa entre la localización de la vegetación y la presencia de embolismo. De 17 pacientes con endocarditis de la válvula mitral, ocho presentaron embolismo (47\%), de 16 pacientes con endocarditis de la válvula aórtica, ocho presentaron embolismo (50\%), de 10 pacientes con endocarditis asociada a cables de dispositivos o catéteres, cuatro embolizaron (40\%) y de seis pacientes con endocarditis de la válvula tricúspide, dos embolizaron (33\%).
Tabla 3. Microorganismo aislado.

\begin{tabular}{|c|c|}
\hline \multicolumn{2}{|l|}{ Microbiología } \\
\hline Staphylococcus & $19(39.6)$ \\
\hline Staphylococcus aureus meticilino sensible & 13 \\
\hline Staphylococcus aureus meticilino resistente & 3 \\
\hline CNS & 3 \\
\hline Enterococcus & $2(4.1)$ \\
\hline 1: E. faecalis & 2 \\
\hline 2: E.faecium & 0 \\
\hline Streptococcus & $7(14.5)$ \\
\hline Streptococcus viridans & 1 \\
\hline Streptococcus bovis & 0 \\
\hline Otras especies de Streptococci & 1 \\
\hline Streptococcus gallolyticus & 1 \\
\hline Streptococcus sanguis & 3 \\
\hline Streptococcus mitis & 1 \\
\hline Organismos gram negativos & $2(4.1)$ \\
\hline Brucella & 0 \\
\hline Pseudonomas aeruginosas & 0 \\
\hline Grupo HACEK & 0 \\
\hline Serratia marcescens & 1 \\
\hline Klebsiella pneumoniae & 1 \\
\hline Cándida & $1(2.1)$ \\
\hline Candida albicans & 0 \\
\hline Candida tropicalis & 1 \\
\hline Candida parapsilosis & 0 \\
\hline $\begin{array}{l}\text { CNS: Staphylococcus coagulasa negativo, HAC } \\
\text { Actinobacillus, Cardiobacterium, Eikenella corro }\end{array}$ & influenzae, \\
\hline
\end{tabular}

En los pacientes con vegetaciones móviles se encontró una tendencia a presentar más complicaciones, aunque la asociación no fue estadísticamente significativa, excepto para la presentación de falla cardiaca $(\mathrm{p}=0.04)$ (Tabla 6). De ocho pacientes que presentaron embolismo a SNC, siete $(87.5 \%)$ tenían vegetaciones móviles y solo un paciente tenía vegetaciones no móviles. De nueve pacientes que presentaron embolismo a pulmón, ocho (88.8\%) tenían vegetaciones móviles y solo uno tenía vegetaciones no móviles. El 100\% de los pacientes que presentaron perforación valvular como complicación tenían vegetaciones móviles.

La mayoría de pacientes tenían vegetaciones $\geq 10 \mathrm{~mm}$ de diámetro ( 35 pacientes), de estos el $100 \%$ presentaron embolismo como complicación. De los pacientes con vegetaciones $<10 \mathrm{~mm}$ (10 pacientes) solo tres presentaron embolismo, aunque esta relación no alcanzó a ser estadísticamente significativa. 
Tabla 4. Hallazgos ecocardiográficos

\begin{tabular}{|c|c|}
\hline Vegetaciones, $n(\%)$ & $45(93.7)$ \\
\hline Tamaño máximo en mm ( \pm DS) Número vegetaciones & $17.6(11.3)$ \\
\hline 1 & $24(53.3)$ \\
\hline 2 & $11(24.4)$ \\
\hline 3 & $10(22.2)$ \\
\hline Móviles Localización & $40(88.8)$ \\
\hline Aórtica & $16(35,5)$ \\
\hline Mitral & $17(37.7)$ \\
\hline Tricuspídea & $6(13.3)$ \\
\hline Intracavitarias & $3(6.6)$ \\
\hline Cables de dispositivos o catéteres & $10(22.2)$ \\
\hline Válvula AV común & $1(2.2)$ \\
\hline Pulmonar & $1(2.2)$ \\
\hline Insuficiencia aórtica & $21(43.7)$ \\
\hline Insuficiencia mitral & $29(60.4)$ \\
\hline Insuficiencia tricuspídea & $22(45.8)$ \\
\hline Insuficiencia pulmonar & $3(6.3)$ \\
\hline FEVI\% & $60(46-65)$ \\
\hline PSAP $\mathrm{mmHg}$ & $45(38-58)$ \\
\hline \multicolumn{2}{|c|}{$\begin{array}{l}\text { FEVI: fracción de eyección del ventrículo izquierdo, PSAP: presión sistólica de } 1 \\
\text { arteria pulmonar, AV: aurículo-ventricular }\end{array}$} \\
\hline
\end{tabular}

Se encontró una asociación estadísticamente significativa entre el tamaño de las vegetaciones $(\geq 10 \mathrm{~mm})$ y la presencia de fuga paravalvular $(\mathrm{p}=0.03)$ y dehiscencia de prótesis $(\mathrm{p}=0.03)$.

Dentro de las características basales de los pacientes, la edad avanzada (mayor de 55 años) se asoció de forma estadísticamente significativa con el riesgo de embolismo periférico $(\mathrm{p}=0.04)$; otras variables evaluadas como la presencia de diabetes mellitus, hipertensión arterial o falla

Tabla 6. Movilidad de las vegetaciones y complicaciones.

\begin{tabular}{|l|c|c|c|}
\hline \multicolumn{4}{|c|}{ Vegetaciones y complicaciones } \\
\hline & $\begin{array}{c}\text { Móviles } \\
\mathbf{n}(\%)\end{array}$ & $\begin{array}{c}\text { No móviles } \\
\text { n (\%) }\end{array}$ & Valor de p \\
\hline Embolismo SNC & $7(87.5)$ & $1(12.5)$ & 0.64 \\
\hline Perforación & $11(100)$ & $0(0)$ & 0.22 \\
\hline Fuga paravalvular & $1(100)$ & $0(0)$ & 0.88 \\
\hline Derrame pericárdico & $14(93.3)$ & $1(6.7)$ & 0.45 \\
\hline Falla cardiaca & $20(100)$ & $0(0)$ & 0.04 \\
\hline SNC: sistema nervioso central & & & \\
\hline
\end{tabular}

Tabla 5. Complicaciones de los pacientes con endocarditis infecciosa.

\begin{tabular}{|c|c|}
\hline \multicolumn{2}{|l|}{ Complicaciones } \\
\hline Absceso de la raíz aórtica, $n(\%)$ & $2(4.2)$ \\
\hline Pseudoaneurisma & 0 \\
\hline Aneurisma valvular & 0 \\
\hline Perforación & $11(22.9)$ \\
\hline Fístula & $1(2.1)$ \\
\hline Fuga paravalvular & $2(4.2)$ \\
\hline Dehiscencia de prótesis & $2(4.2)$ \\
\hline Destrucción valvular & 0 \\
\hline Ruptura de cuerdas tendinosas & $3(6.3)$ \\
\hline Derrame pericárdico & $15(31.3)$ \\
\hline Falla cardíaca & $20(41.7)$ \\
\hline Extensión perivalvular & $4(8.3)$ \\
\hline Embolismo & $21(43.7)$ \\
\hline Pulmón & 9 \\
\hline SNC & 8 \\
\hline Extremidades & 6 \\
\hline Bazo & 2 \\
\hline Piel & 2 \\
\hline Espondilodiscitis & 1 \\
\hline Renal & 1 \\
\hline Choque séptico & $13(27.1)$ \\
\hline Bloqueo AV & $1(2.1)$ \\
\hline Falla renal con requerimiento de terapia de remplazo renal & $14(29.2)$ \\
\hline SNC: sistema nervioso central, AV: aurículo-ventricular. & \\
\hline
\end{tabular}

Tabla 7. Factores asociados con mortalidad intrahospitalaria: análisis multivariado.

\begin{tabular}{|l|c|c|c|}
\hline \multicolumn{4}{|c|}{ Análisis multivariado } \\
\hline \multicolumn{1}{|c|}{ Variable } & Valor de p & Exp (B) & 95\% IC \\
\hline Choque séptico & 0.005 & 14.91 & $(2.30-96.49)$ \\
\hline Falla renal con TRR & 0.033 & 6.57 & $(1.16-37.22)$ \\
\hline Staphylococcus & 0.048 & 5.11 & $(1.01-25.79)$ \\
\hline Inmunosupresores & 0.047 & 9.37 & $(1.03-85.24)$ \\
\hline \multicolumn{5}{|c|}{ TRR: terapia de reemplazo renal. } \\
\hline
\end{tabular}


renal no se asociaron de forma significativa con la presencia de embolismo.

La falla cardiaca se presentó como complicación en 20 pacientes, 19 con endocarditis de válvula nativa, de estos, 11 pacientes con vegetación de la válvula aórtica y ocho pacientes con vegetación de la válvula mitral.

Se presentaron dos casos de absceso de la raíz aórtica en pacientes con endocarditis de válvula nativa, en uno el aislamiento microbiológico fue un E. faecalis y en el otro un S. lugdunensis.

La extensión perivalvular se presentó en cuatro pacientes con endocarditis de válvula nativa, con vegetaciones localizadas en la válvula aórtica (2), tricuspídea (1) e intracavitarias (1); se aisló Staphylococcus en tres pacientes y $E$. faecalis en un paciente.

Se encontraron 11 casos de perforación valvular, siete con compromiso de la válvula mitral y cuatro de la válvula aórtica. El germen más comúnmente implicado en estos casos fue el S. aureus $(36.4 \%)$.

Usando un análisis multivariado se encontró una asociación significativa entre mortalidad como desenlace y la presencia de choque séptico, falla renal, infección por Staphylococcus y uso de inmunosupresores. No se encontró asociación con otras variables evaluadas (Tabla 7).

\section{Discusión}

Aunque la incidencia de la EI en la población general no fue analizada, los resultados indican que a pesar de los avances en el cuidado de la salud la EI sigue siendo una enfermedad que impone un reto diagnóstico y terapéutico para los médicos, especialmente ahora que el patrón de presentación difiere sustancialmente del de hace algunos años. La población en mayor riesgo de infección ya no son los pacientes jóvenes con enfermedad valvular reumática conocida y ahora son personas de edad más avanzada sin enfermedad valvular aparente $(5,8)$. En el presente estudio la mayoría de pacientes no tenían una condición cardiaca predisponente de base (62.5\%), al igual que lo reportado por Castillo y colaboradores en un estudio epidemiológico en España, donde en los últimos siete años el 64\% de los casos de EI eran pacientes sin enfermedad cardiaca predisponente (5).

La edad promedio de presentación fue 53.8 \pm 19.2 años. $\mathrm{El}$ aumento en la edad de los pacientes con EI coincide con otros estudios realizados en la última década $(9,10)$. Esto puede ser explicado por la mayor sobrevida de la población y por el aumento del número de casos de EI asociada a hospitalización y al cuidado de la salud. De acuerdo con la gran mayoría de los estudios, encontramos predominio de la afectación en hombres con una relación hombre: mujer de 1.5:1 $(5,6,11)$.

Nuestro estudio confirmó muchas características clínicas de la endocarditis que ocurren en otras partes del mundo. Por ejemplo, la mayoría de los casos de EI fueron en válvula nativa $(75 \%)$ y la válvula mitral fue la más comúnmente afectada, seguida por la válvula aórtica $(10,11)$.
La EI con hemocultivos negativos puede ocurrir hasta en $31 \%$ de los casos de EI y a menudo impone un reto considerable para el diagnóstico y tratamiento, ocurre más comúnmente como consecuencia del uso previo de antibióticos, resaltando la necesidad de suspender los antibióticos y repetir los hemocultivos en esta situación (12). En el presente estudio encontramos una prevalencia de EI con hemocultivos negativos del $37.5 \%$.

Los estudios publicados difieren con respecto a cuál es el microorganismo causante más frecuente, esta variación puede estar en relación a la población estudiada $(10,11,14$, 15). Las series que incluyen pacientes con uso de drogas intravenosas reportan mayor prevalencia de $S$. aureus como agente causante, otras series incluyen predominantemente pacientes con EI de válvula protésica. Según lo reportado en algunos estudios recientes, las especies de Staphylococcus han superado a los Streptococcus como microorganismos causantes más frecuente de EI de válvula nativa $(12,13)$. En el presente estudio, que incluyó predominantemente casos de EI de válvula nativa, sin encontrar casos de uso de drogas intravenosas, las especies de Staphylococcus fueron los microorganismos más frecuentes (39.6\%), especialmente el Staphylococcus aureus (33\%), seguidos por las especies de Streptococcus (14.5\%), indicando que el viraje informado a nivel mundial de $S$. viridans como principal germen en el pasado a $S$. aureus actualmente, también se cumple en la población evaluada y probablemente esté en relación a las mismas razones, como aumento de procedimientos invasivos (catéteres vasculares, dispositivos intracardiacos, etc).

Los síntomas de presentación más frecuentes fueron fatiga y fiebre, sin embargo la prevalencia de fiebre en nuestro estudio fue menor a lo reportado por otros autores (12). El $29.2 \%$ de los pacientes no reportaron fiebre, lo cual sugiere que la ausencia de fiebre no debe excluir el diagnóstico de EI en un paciente con características clínicas sugestivas.

La ausencia de soplo en pacientes con EI ha sido descrita previamente, sin embargo la proporción de casos sin soplo $(52.1 \%)$ fue mucho mayor en nuestro estudio que lo reportado en un estudio retrospectivo por Fefer P. y col. (16).

La ecocardiografía fue el pilar fundamental para el diagnóstico de EI en nuestra serie. Se detectaron vegetaciones en la mayoría de los casos (93.7\%). El tamaño de las vegetaciones $(17.6 \pm 11.3 \mathrm{~mm})$ fue mucho mayor en nuestro estudio a lo reportado en un registro español por Roca B. et al. (17), donde la longitud promedio fue de $9 \mathrm{~mm}$. La insuficiencia mitral fue la condición cardiaca predominante en nuestro estudio (60.4\%) similar a lo reportado por Winston y Bolger (18).

La prevalencia de embolismo fue alta en nuestro estudio (43.7\%) coincidiendo con lo publicado previamente por Vilacosta y col. (19). Los sitios más frecuentes de embolismo fueron el pulmón (42.8\%) y el sistema nervioso central (38\%). La prevalencia encontrada de embolismo al bazo fue mucho menor a lo reportado en la literatura y esto puede reflejar un subdiagnóstico por menor realización 
de estudios de imagen de abdomen de rutina en estos pacientes (12).

Varios estudios observacionales han evaluado la asociación entre la localización de las vegetaciones por ecocardiograma y el riesgo de complicaciones, (19-21), encontrando la localización de las vegetaciones en la válvula mitral como un factor de riesgo para embolismo. En el presente estudio no se encontró una asociación estadísticamente significativa entre la localización de la vegetación y la presencia de embolismo, con similar proporción de embolismo en pacientes con vegetaciones localizadas en la válvula mitral y aórtica. Las vegetaciones móviles se asociaron con mayor incidencia de complicaciones como embolismo, aunque esta asociación no alcanzó a ser estadísticamente significativa en nuestro estudio, tal vez debido al tamaño de la muestra.

Como se mencionó anteriormente el tamaño de las vegetaciones en este estudio es mucho mayor al reportado en otras series, las vegetaciones $\geq 10 \mathrm{~mm}$ presentaron con mayor frecuencia embolismo como complicación.

El análisis multivariado reveló un aumento del riesgo de muerte por endocarditis con la presencia de choque séptico, falla renal, infección por Staphylococcus y uso de inmunosupresores. Otros estudios en este aspecto han llegado a conclusiones similares a las nuestras con respecto a los factores asociados con mayor mortalidad (22-25). Sin embargo también hay estudios que han encontrado asociación significativa de otros factores con mortalidad, como falla cardiaca y edad avanzada (26). Las discrepancias pueden explicarse por las diferencias en el diseño de los estudios y la falta de uniformidad en las variables analizadas.

Nuestro estudio tiene varias limitaciones, particularmente el tamaño de la muestra, el diseño retrospectivo del mismo y la baja realización de pruebas serológicas y moleculares que limitan la detección de organismos fastidiosos como Brucella, Bartonella, Coxiella y otras causas raras de EI que no se pueden descartar con los estudios microbiológicos tradicionales.

Este estudio aporta un perfil epidemiológico de la EI en Medellín-Colombia. Si bien esta serie incluyó 48 pacientes en un periodo de tres años en un hospital de tercer nivel, esta es una frecuencia comparable o mayor a la reportada por otras series, como la de Senior (27) en la que se incluyeron 92 pacientes en un periodo mayor (11 años) y a partir de tres centros de referencia y la de Olaya Sánchez y col. (28) con 34 pacientes en cuatro años. Los tamaños de estas series se explican por la baja prevalencia de la endocarditis infecciosa.

\section{Conclusiones}

El presente estudio muestra las características epidemiológicas, clínicas y ecocardiográficas de una población con EI en un hospital de tercer nivel de Medellín- Colombia. En nuestra población la EI actualmente es una enfermedad que afecta predominantemente personas de edad avanzada sin enfermedad cardiaca predisponente de base. La mayoría de los casos corresponden a endocarditis de válvula nativa con mayor compromiso de la válvula mitral. El S. aureus fue el microorganismo causante más frecuente. La ecocardiografía es el pilar fundamental para el diagnóstico. Este estudio ayuda en el reconocimiento de factores pronóstico que identifican pacientes con mayor riesgo de mortalidad intrahospitalaria en nuestra población.

\section{Fuente de Apoyo}

Universidad CES.

\section{Agradecimientos}

Agradecimientos especiales a la Dra. Elsa María Vásquez Trespalacios por su colaboración con el análisis estadístico de este estudio. A la Universidad CES y al Hospital General de Medellín.

\section{Referencias}

1. Mylonakis E, Calderwood SB. Infective endocarditis in adults. $N$ Engl $\mathrm{J} \mathrm{Med}$ 2001;345: 1318-30.

2. Netzer RO, Zollinger E, Seiler C, Cerny A. Infective endocarditis: Clinical spectrum, presentation and outcome. An analysis of 212 cases 1980-1995. Heart 2000;84:25-30.

3. Tleyjeh IM, Steckelberg JM. Changing Epidemiology of Infective Endocarditis Curr Infect Dis Reports 2006;8:265-70.

4. Griffin MR, Wilson WR, Edwards WD, O'Fallon WM, Kurland LT. Infective endocarditis: Olmsted County. Minnesota. JAMA 1985;254: 1199-202.

5. Castillo JC, Anguita M, Ruiz M, Pena L, Santisteban M. y col. Changing Epidemiology of Native Valve Infective Endocarditis. Rev Esp Cardiol. 2011;64(7):594-598

6. Hill EE, Herijgers P, Claus P, Vanderschueren S, Herregods MC, Peetermans WE. Infective endocarditis: changing epidemiology and predictors of 6-month mortality: a prospective cohort study. Eur Heart J. 2007;28:196-203.

7. Cabell CH, Jollis JG, Peterson GE, Corey GR, Anderson DJ, Sexton DJ, et al. Changing patient characteristics and the effect on mortality in endocarditis. Arch Intern Med. 2002;162:90-4.

8. Strom BL, Abrutyn E, Berlin JA, Kinman JL, Feldman RS, Stolley PD, et al. Dental and Cardiac Risk Factors for Infective Endocarditis. A Population-Based, Case-Control Study. Ann Intern Med 1998;129: 761-9.

9. Tuğcu A, Yıldırımtürk Ö, Baytaroğlu C, Kurtoğlu H, Köse Ö, Şener M, et al. Clinical spectrum, presentation, an risk factors for mortality in infective endocarditis: A review of 68 cases at a tertiary care center in Turkey. Turk Kardiyol Dern Ars 2009;37: 9-18.

10. Murdoch DR, Corey GR, Hoen B, Miro JM, Fowler VG, Bayer AS, et al. Clinical presentation, etiology, and outcome of infective endocarditis in the 21 st century. Arch Intern Med 2009;169: 463-73.

11. Ferreiros E, Nacinovich F, Casabe JH, Modenesi JC, Swieszkowski S, Cortl C, et al. Epidemiologic, clinical and microbiologic profile of infective endocarditis in Argentina: a national survey. The Endocarditis Infecciosa en la Republica Argentina-2 Study. Am Heart J. 2006;151:545-52.

12.Habib G, Lancellotti P, Antunes MJ, Bongiorni MG, Casalta JP, Del Zotti F, et al. 2015 ESC Guidelines for the management of infective endocarditis. The Task Force for the Management of Infective Endocarditis of the European Society of Cardiology (ESC). European Heart Journal. First published online: 29 August 2015

13. Barrau K, Boulamery A, Imbert G, Casalta JP, Habib G, Messana T, et al. Causative organisms of infective endocarditis according to host status. Clin Microbiol Infect 2004; 10: 302-8.

14. Tleyjeh IM, Steckelberg JM, Murad HS, Anavekar NS, Ghomrawi HM, Mirzoyev Z, et al. Temporal trends in infective endocarditis. A population-based study in Olmsted County. JAMA. 2005;293: 3022-8.

15. Hoen B, Alla F, Selton-Suty C, Beginot I, Bouvet A, Briancon S, et al. Changing profile of infective endocarditis: results of a 1-year survey in France. JAMA. 2002;288: 75-81.

16. Fefer P, Raveh D, Rudensky B, Schlesinger Y, Yinnon AM. Changing epidemiology of infective endocarditis: a retrospective survey of 108 cases, 1990-1999. Eur J Clin Microbiol Infect Dis 2002;21:432-7.

17. Roca B, Marco JM. Presentation and outcome of infective endocarditis in Spain: a retrospective study. International Journal of Infectious Diseases (2007) 11, 198203

18. Winston LG, Bolger AF. Modern epidemiology, prophylaxis, and diagnosis and 
therapy for infective endocarditis. Curr Cardiol Rep 2006;8:102-8.

19. Vilacosta I, Graupner C, San Roman JA, Sarria C, Ronderos R, Fernandez C, Mancini L, Sanz O, Sanmartin JV, Stoermann W. Risk of embolization after institution of antibiotic therapy for infective endocarditis. J Am Coll Cardiol 2002;39:1489-1495.

20. Rohmann S, Erbel R, Gorge G, Makowski T, Mohr-Kahaly S, Nixdorff U, Drexler M, Meyer J. Clinical relevance of vegetation localization by transoesophageal echocardiography in infective endocarditis. Eur Heart J 1992;13:446-452.

21. Sanfilippo AJ, Picard MH, Newell JB, Rosas E, Davidoff R, Thomas JD, Weyman AE. Echocardiographic assessment of patients with infectious endocarditis: prediction of risk for complications. J Am Coll Cardiol 1991;18:1191-1199

22. Leone S, Ravasio V, Durante-Mangoni E, Crapis M, Carosi G, Scotton PG, Barzaghi, N, Falcone M, Chinello P, Pasticci MB, Grossi P, Utili R, Viale P, Rizzi M, Suter F. Epidemiology, characteristics, and outcome of infective endocarditis in Italy: the Italian Study on Endocarditis. Infection 2012;40:527-535.

23. Olmos C, Vilacosta I, Fernandez C, Lopez J, Sarria C, Ferrera C, Revilla A, Silva J, Vivas D, Gonzalez I, San Roman JA. Contemporary epidemiology and prognosis of septic shock in infective endocarditis. Eur Heart $J$ 2013;34:1999_ 2006.

24. Chu VH, Cabell CH, Benjamin Jr DK, Kuniholm EF, Fowler Jr VG, Engemann J, et al. Early predictors of in-hospital death in infective endocarditis. Circulation 2004;109:1745-9.

25. Casabe JH, Deschle H, Cortes C, Stutzbach P, Hershson A, Nagel C, et al. Predictors of hospital mortality in 186 cases of active infective endocarditis treated in a tertiary medical center (1992-2001). Rev Esp Cardiol 2003;56:578-85.

26. Netzer R, Altwegg SC, Zollinger S, Täuber M, Carrel T, Seiler C. Infective endocarditis: determinants of long term outcome. Heart 2002;88:61-66

27. Senior JM. Endocarditis infecciosa. Descripción clínico-epidemiológica. Acta Med Colomb 1995; 20(4):169-75

28. Olaya Sánchez A, Vargas Vergara D, Montes Zabala L, Avila Cortéz Y, Cárcamo Molina LM. Descripción clínica, microbiológica y ecocardiográfica de la endocarditis infecciosa en un hospital de Bogotá durante los años 2013-2017. Acta Med Colomb 2019; 44(4) 